\title{
Impact of Symmetrical Phase Shifter on Power-System State Estimation
}

\author{
Tomasz Okon, Kazimierz Wilkosz \\ Department of Electrical Power Engineering, Wroclaw University of Science and Technology, \\ Wybrzeze Wyspianskiego 27, 50-370 Wroclaw, Poland \\ tomasz.okon@pwr.edu.pl
}

\begin{abstract}
The paper deals with Power System State Estimation (PSSE) that plays essential role in modern dispatcher centres. The aim of the paper is to present results of original investigations on change of properties of PSSE when a symmetrical phase shifter is installed in a Power System (PS). The mentioned phase shifter is one of the types of phase shifters. Such the phase shifter is installed in the Polish Power System. In the paper, the following indices characterizing properties of PSSE are considered: the number of iterations in PSSE calculation, the conditionality of the solved equations and accuracy of estimation. The investigations are made using the IEEE 14-bus test system. The properties of PSSE are analysed when there is the phase shifter and when there is not such the device in PS for the polar and rectangular coordinate systems. The investigations are so organized to take into account possibly all space of states of PS. Results of the investigations show that there is noticeable change of the properties of PSSE as an effect of installation of the phase shifter in PS. In the paper, also such the cases are indicated, in which those changes are the smallest.
\end{abstract}

Index Terms-Model; Phase shifter; Power flow control; Power system; State estimation.

\section{INTRODUCTION}

One of devices, which are utilized for power flow control in modern Power Systems (PSs), is a Phase Shifter (PhS). Its characteristic feature is possibility of control of phase angles of voltages in PS. Changing the phase shift between the terminal voltages of a power line implies changes of the power flow through this line [1]-[3]. In consequence, $\mathrm{PhS}$ changes power flows in PS, what can lead to elimination of undesirable power flows.

Presence of PhS in PS should be taken into account in calculations made for this system, in particular, in State Estimation (SE) [4]. The Power-System State Estimation (PSSE) is important part of the real-time PS modelling [5], which should ensure the reliable estimate of the PS state vector.

The paper presents results of original investigations on change of properties of PSSE when PhS is installed in PS. The considered type of PhS is a symmetrical one [1]-[3]. A symmetrical $\mathrm{PhS}$ create an output voltage with an altered phase angle compared to the input voltage, but with the same magnitude [3]. In this way one forms the voltage at the beginning of a power line, of which phase is different than

Manuscript received 11 November, 2017; accepted 17 April, 2018. the phase of the voltage before $\mathrm{PhS}$.

There is relatively small number of papers presenting the problem of evaluation of properties of SE realized for PS with $\mathrm{PhS}$ [6], [7]. In [6], [7], the following indices characterizing properties of SE are considered: the number of iterations in PSSE calculation, the conditionality of the solved equations and accuracy of estimation. In [6], it is assumed that $\mathrm{PhS}$ is modelled as ideal transformer with a complex tap ratio in series with two-port $\pi$ circuit which represents transformer losses. PSSE is considered in the polar and rectangular coordinate systems. [7] is devoted to one of the type of PhSs, which is a quadrature booster. In [7], the results of investigations of PSSE in the rectangular coordinate system are presented. In [7], significance of specific information on the quadrature booster from the point of view of properties of PSSE is investigated.

This paper contains the results of a next part of the investigations of the authors to be focused on properties of $\mathrm{SE}$ realized for PS with the symmetrical PhS. One takes into account such indices characterizing properties of $\mathrm{SE}$ as in [6], [7]. In this paper, PSSE is considered in the rectangular and polar coordinate systems. Up to now, such the investigations have not been considered in existing papers. It should be underlined that the mentioned investigations are essential from the point of view of SE realized for PS in which there is the above-pointed PhS. Such situation is in the Polish Power System. Now, in that system there are the symmetrical PhSs. Knowledge of properties of different solutions of SE for PS with PhS enables to choose the best one for the aim of the real-time PS modelling in dispatch centre.

In the paper, the symmetrical $\mathrm{PhS}$ is modelled with use of two real voltage sources. One of them is in a shunt branch and second one is in a series branch. The assumed model is other than models of PhSs that can be found in other papers [2], [8], [9]. The here-considered model can be used for modelling different types of $\mathrm{PhSs}$, otherwise than models from the earlier-mentioned papers.

In the further part of the paper, the considered method for PSSE and indices characterizing its properties are presented. Next, a model of the symmetrical PhS is formulated. A main part of the paper is description of the investigations, whose aim is to show differences between properties of SE for PS with the symmetrical $\mathrm{PhS}$ and without this device. At the end, the most important conclusions from the conducted 
investigations are given.

\section{COnsidered State Estimation Method}

\section{A. An Idea of the Method}

In the described investigations, the weighted least squares PSSE method is considered. An objective function of that method can be written as follows [4]

$$
J(\mathbf{x})=\frac{1}{2}[\mathbf{z}-\mathbf{h}(\mathbf{x})]^{\mathbf{T}} \mathbf{R}^{-\mathbf{1}}[\mathbf{z}-\mathbf{h}(\mathbf{x})]
$$

where: $\mathbf{x}$ is a PS state vector; $\mathbf{z}$ is a vector of measurements; $\mathbf{h}(\mathbf{x})$ is a vector of functions (also nonlinear), representing dependence of measured quantities from the state vector $\mathbf{x}$; $\mathbf{R}$ is a diagonal matrix of measurement covariances.

State vector $\mathbf{x}$ in the rectangular coordinate system is defined as $\mathbf{x}=\left[e_{1}, e_{2}, \ldots, e_{n}, f_{2}, f_{3}, \ldots, f_{n}\right]^{\mathrm{T}}$, where: $e_{i} i=1,2$, $\ldots, n$ are real parts of voltages at buses $1,2, \ldots, n ; f_{i} i=2,3$, $\ldots, n$ are imaginary parts of voltages at buses $2,3, \ldots, n$. State vector $\mathbf{x}$ in the polar coordinate system is defined as $\mathbf{x}=\left[\delta_{2}, \delta_{3}, \ldots, \delta_{n}, V_{1}, V_{2}, \ldots, V_{n}\right]^{\mathrm{T}}$, where: $\delta_{i} i=2,3, \ldots, n$ phase angles of voltages at buses $2,3, \ldots, n ; V_{i} i=1,2, \ldots, n$ - magnitudes of voltages at buses $1,2, \ldots, n$.

The elements of $\mathbf{h}(\mathbf{x})$ can be determined as follows [10]:

$$
\begin{gathered}
V_{i}=V_{i}, \\
P_{i}-j Q_{i}=\overline{\mathbf{V}}_{i}^{*} \mathbf{Y}_{\text {rowi }} \mathbf{V}, \\
P_{i j}-j Q_{i j}=\left[\begin{array}{cc}
-\left(\overline{\mathbf{y}}_{s i}+\overline{\mathbf{y}}_{i j}\right) & \overline{\mathbf{y}}_{i j}
\end{array}\right] \cdot\left[\begin{array}{ll}
V_{i}^{2} & \overline{\mathbf{V}}_{j} \cdot \overline{\mathbf{V}}_{i}^{*}
\end{array}\right]^{T},
\end{gathered}
$$

where $P_{i}, Q_{i}$ are an active and reactive power injection at $i$-th bus, respectively; $P_{i j}, Q_{i j}$ are an active and reactive power flow, respectively, between $i$-th and $j$-th bus, measured at $i$-th bus; $\overline{\mathbf{V}}_{i}$ is a voltage at $i$-th bus; $\overline{\mathbf{y}}_{i j}$ is an admittance of the series branch connecting $i$-th and $j$-th bus; $\overline{\mathbf{y}}_{s i}$ is an admittance of the shunt branch at $i$-th bus; $\mathbf{Y}_{\text {row } i}$ is $i$-th row of an admittance matrix $\mathbf{Y}_{\text {row } i}=\left[\overline{\mathbf{Y}}_{i 1}, \overline{\mathbf{Y}}_{i 2}, \ldots, \overline{\mathbf{Y}}_{i n}\right], \overline{\mathbf{Y}}_{i k} i$, $k=1,2, \ldots, n$ are elements of the admittance matrix; $\mathbf{V}$ is a vector $\mathbf{V}=\left[\overline{\mathbf{V}}_{1}, \overline{\mathbf{V}}_{2}, \ldots, \overline{\mathbf{V}}_{n}\right]^{\mathrm{T}}$.

In the described investigations, it is assumed that a solution of the PSSE problem is achieved by solving the normal-equation set

$$
\mathbf{G}\left(\mathbf{x}^{k}\right) \times\left(\mathbf{x}^{k+1}-\mathbf{x}^{k}\right)=-\mathbf{g}\left(\mathbf{x}^{k}\right),
$$

where $k$ is a number of iteration, $\mathbf{x}^{k}$ is a solution vector at $k$-th iteration, $\quad \mathbf{G}\left(\mathbf{x}^{k}\right)=\mathbf{H}^{T}\left(\mathbf{x}^{k}\right) \times \mathbf{R}^{-1} \times \mathbf{H}\left(\mathbf{x}^{k}\right)$, $\mathbf{H}(\mathbf{x})=\frac{\partial \mathbf{h}(\mathbf{x})}{\partial \mathbf{x}}, \mathbf{g}(\mathbf{x})=\frac{\partial J(\mathbf{x})}{\partial \mathbf{x}}=-\mathbf{H}^{T}(\mathbf{x}) \mathbf{R}^{-1}[\mathbf{z}-\mathbf{h}(\mathbf{x})]$.

$\mathbf{G}(\mathbf{x})$ is called as a gain matrix, to be a symmetric, sparse, and positive determined matrix for a fully-observable PS.

\section{B. Indices Characterising the Method}

In the paper, properties of PSSE are characterized by the following indices [10]: (i) number of iterations in calculation process $\left(n_{i t}\right)$, (ii) a condition number of the gain matrix (cond $(\mathbf{G}))$, (iii) ratio $J_{e} / J_{m}\left(A=J_{e} / J_{m}\right)$.

Number of iterations $n_{i t}$ affects the time of calculations.

Condition number cond $(\mathbf{G})$ is a measure of conditioning of a SE process [11]. In the paper, the following definition of condition number $\operatorname{cond}(\mathbf{G})$ is taken into account: $\operatorname{cond}(\mathbf{G})=\lambda_{M} / \lambda_{m}$, where $\lambda_{m}, \lambda_{M}$ are the minimal and maximal eigenvalues of matrix $\mathbf{G}(\mathbf{x})$, respectively.

The larger the condition number is, the worse the conditioning of the estimation process is. The illconditioning of the SE process often leads to a worse convergence of the SE process or even to lack of the convergence of this process.

In the ratio $A=J_{e} / J_{m}, J_{e}$ and $J_{m}$ are calculated as $J_{e}=\frac{1}{m} \sum_{i=1}^{m}\left[\left(\hat{z}_{i}-z_{i}^{r}\right) / \sigma_{i}\right]^{2}$ and $J_{m}=\frac{1}{m} \sum_{i=1}^{m}\left[\left(z_{i}-z_{i}^{r}\right) / \sigma_{i}\right]^{2}$ where $z_{i}, \hat{z}_{i}, z_{i}^{r}$ are the measured, estimated and real values of $i$-th measured quantity, respectively; $\sigma_{i}$ is a variance of the measurement of $i$-th quantity; $m$ is a number of the measured quantities. Ratio $A$ characterizes accuracy of SE [12]. That ratio should satisfy the condition $A<1$. If that condition is not satisfied, then accuracy of results of PSSE is assessed as insufficient.

The smaller the distinguished indices, the better the properties of SE are.

\section{DESCRIPTION OF PHASE SHIFTER}

An equivalent circuit of $\mathrm{PhS}$ is shown in Fig. 1. In that circuit, there are: a shunt Excitation Transformer (ET), a series Boosting Transformer (BT), which injects a series voltage $\left(\overline{\mathbf{V}}_{\mathrm{BT}}\right)$ in PS, and a tap changer. Voltage $\overline{\mathbf{V}}_{\mathrm{BT}}$ is controlled by the tap changer.

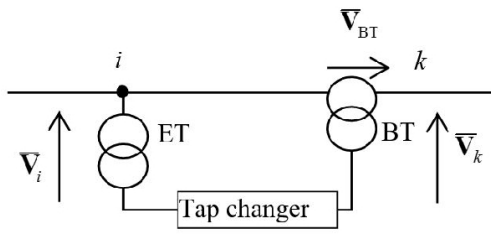

Fig. 1. An equivalent circuit of the phase shifter.

A symmetrical $\mathrm{PhS}$ create an output voltage whose magnitude is equal to the magnitude of voltage $\overline{\mathbf{V}}_{i}$. The phasor diagram for the symmetrical $\mathrm{PhS}$ is in Fig. 2.

In the carried out investigations, $\mathrm{PhS}$ is represented by the model, to be shown in Fig. 3, containing two controllable voltage sources with internal impedances $\overline{\mathbf{z}}_{\mathrm{ET}}$ and $\overline{\mathbf{z}}_{\mathrm{BT}}$, which represent impedances of ET and BT, respectively.

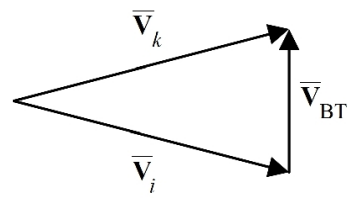

Fig. 2. Phasor diagram for the symmetrical phase shifter. 


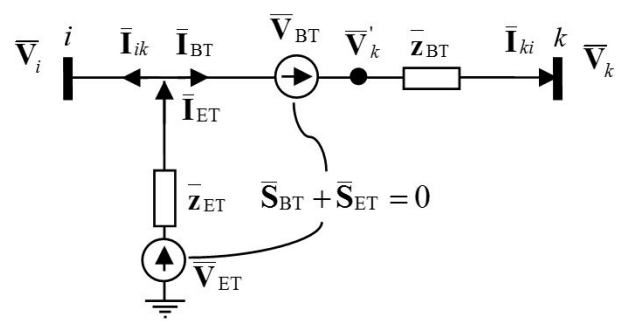

Fig. 3. The assumed model for the considered phase shifter.

For the considered model, the following equations can be derived:

$$
\begin{gathered}
\overline{\mathbf{S}}_{i k}^{*}=-V_{i}^{2}\left(\overline{\mathbf{y}}_{\mathrm{ET}}+\overline{\mathbf{y}}_{\mathrm{BT}}\right)+ \\
+\overline{\mathbf{V}}_{k} \overline{\mathbf{V}}_{i}^{*} \overline{\mathbf{y}}_{\mathrm{BT}}-\overline{\mathbf{V}}_{\mathrm{BT}} \overline{\mathbf{V}}_{i}^{*-} \overline{\mathbf{y}}_{\mathrm{BT}}+\overline{\mathbf{V}}_{\mathrm{ET}} \overline{\mathbf{V}}_{i}^{*-} \overline{\mathbf{y}}_{\mathrm{BT}}, \\
\overline{\mathbf{S}}_{k i}^{*}=-V_{k}^{2} \overline{\mathbf{y}}_{\mathrm{BT}}+\overline{\mathbf{V}}_{i} \overline{\mathbf{V}}_{k}^{*} \overline{\mathbf{y}}_{\mathrm{BT}}+\overline{\mathbf{V}}_{\mathrm{BT}} \overline{\mathbf{V}}_{k}^{*} \overline{\mathbf{y}}_{\mathrm{BT}}, \\
\overline{\mathbf{S}}_{\mathrm{BT}}^{*}=V_{\mathrm{BT}}^{2} \overline{\mathbf{y}}_{\mathrm{BT}}+\overline{\mathbf{V}}_{i} \overline{\mathbf{V}}_{\mathrm{BT}}^{*} \overline{\mathbf{y}}_{\mathrm{BT}}-\overline{\mathbf{V}}_{k} \overline{\mathbf{V}}_{\mathrm{BT}}^{*} \overline{\mathbf{y}}_{\mathrm{BT}}, \\
\overline{\mathbf{S}}_{\mathrm{ET}}^{*}=V_{\mathrm{ET}}^{2} \overline{\mathbf{y}}_{\mathrm{ET}}-\overline{\mathbf{V}}_{i} \overline{\mathbf{V}}_{\mathrm{ET}}^{*} \overline{\mathbf{y}}_{\mathrm{ET}},
\end{gathered}
$$

where $\overline{\mathbf{y}}_{\mathrm{ET}}=1 / \overline{\mathbf{z}}_{\mathrm{ET}}, \overline{\mathbf{y}}_{\mathrm{BT}}=1 / \overline{\mathbf{z}}_{\mathrm{BT}}, V_{\mathrm{ET}}, \quad V_{\mathrm{BT}}$ are voltage magnitudes of $\overline{\mathbf{V}}_{\mathrm{ET}}$ and $\overline{\mathbf{V}}_{\mathrm{BT}}$, respectively.

One can state that $\mathrm{PhS}$ cannot absorb and injects complex power, i.e. the complex power supplied to transformer ET equals to the complex power demanded by transformer BT

$$
\overline{\mathbf{S}}_{\mathrm{ET}}+\overline{\mathbf{S}}_{\mathrm{BT}}=0 \text {. }
$$

The powers at buses $i$ and $k$ are now determined as follows:

$$
\left\{\begin{array}{l}
\overline{\mathbf{S}}_{i}=\overline{\mathbf{S}}_{i-\mathrm{AC}}-\overline{\mathbf{S}}_{i k}, \\
\overline{\mathbf{S}}_{k}=\overline{\mathbf{S}}_{k-\mathrm{AC}}-\overline{\mathbf{S}}_{k i},
\end{array}\right.
$$

where $\overline{\mathbf{S}}_{i-\mathrm{AC}}$ and $\overline{\mathbf{S}}_{k-\mathrm{AC}}$ are bus powers determined by formula (3) for buses $i$ and $k$, respectively, when there is no $\mathrm{PhS}$ in PS.

For the symmetrical $\mathrm{PhS}$ the following assumption should be taken into account:

- for the polar coordinate system

$$
V_{i}-V_{k}=0,
$$

- for the rectangular coordinate system

$$
e_{i}^{2}+f_{i}^{2}-e_{k}^{2}-f_{k}^{2}=0 \text {. }
$$

The above assumptions apply to an ideal symmetrical $\mathrm{PhS}$. In practice, relations (12) and (13) are fulfilled only when $\mathrm{PhS}$ is not loaded. Therefore, for the real symmetrical $\mathrm{PhS}$, the following assumptions must be taken into account:

$$
t_{i k}=\frac{V_{i}}{\left|\overline{\mathbf{V}}_{i}+\overline{\mathbf{V}}_{B T}\right|}=1,
$$

and

$$
\alpha_{i k}=\angle\left(\overline{\mathbf{V}}_{i}\right)-\angle\left(\overline{\mathbf{V}}_{i}+\overline{\mathbf{V}}_{B T}\right)
$$

\section{INVESTIGATIONS}

It is not possible to perform analytical investigation of the considered indices characterizing properties of SE in all space of operational states of PS. In this situation, there were carried out: (i) original calculations of the considered indices for representative operational states of the test PS when SE is performed in the rectangular and polar coordinate systems and in PS there is and there is no PhS, (ii) original statistical analyses of the calculated indices, (iii) original discussion on the causes of observed regularities.

\section{A. Assumptions}

The investigations are so organized to ensure representativeness of the considered cases of realization of SE for a test system.

1. The IEEE 14-bus test system (Fig. 4) is used.

2. One considers the test system without and with the real symmetrical $\mathrm{PhS}$.

3. If the $\mathrm{PhS}$ is in the test system, then it is on the line between bus 5 and bus 4 , at bus 5 .

4. One considers PSSE in the rectangular and polar coordinate systems. SE_R_S and SE_P_S stand for SE for the test system with $\mathrm{PhS}$ in the first and second case, respectively. SE_R_S- and SE_P_S- stand for SE for the test system without $\mathrm{PhS}$ in the rectangular and polar coordinate systems, respectively.

5. Each of impedances $\overline{\mathbf{z}}_{\mathrm{ET}}$ and $\overline{\mathbf{z}}_{\mathrm{BT}}$ in the model of $\mathrm{PhS}$ is equal to $0.01+\mathrm{j} 0.1 \mathrm{pu}$.

6. 11 load variants are considered. For the given variant, each active and reactive load and also power injection is defined as $W=0.5 W_{b}+l \times W_{b}$, where $W, W_{b}$ are the calculated and base values of the mentioned quantity; $l \in\{0,0.1,0.2, \ldots, 1\} . V^{0.5+l}$ stands for the variant associated with $l$.

7. For each load variant, the phase shift introduced by $\mathrm{PhS}$ is in the range $\left[-20^{\circ}, 20^{\circ}\right]$ (as it is for Polish $\mathrm{PhSs}$ ).

8. One takes into account the following numbers of Measurement Data (MD): $m_{1}=34, m_{2}=53, m_{3}=68$ and $m_{4}=104$, respectively.

9. For each MD number, 100 locations of measurement systems is randomly generated.

10. Each item of MD is burden with a small error characterized by the Gaussian distribution with a mean equal to zero and standard deviation $\sigma$, defined as [13], [14]: $\quad \sigma=1 / 3[(0.001+0.0025) F S+0.02 M]$ for active power, $\sigma=1 / 3[(0.001+0.005) F S+0.02 M]$ for reactive power, and $\sigma=1 / 3[(0.0005+0.0025) F S+0.003 M]$ for voltage magnitude, where $F S$ is a measurement scope, $M$ is a measured value.

11. To determine differences between properties of SE_P_S and SE_P_S- or SE_R_S and SE_R_S-, the investigations of these SEs are made for the same load variants, MD numbers, locations of measurement systems and characteristics of small errors burdening MD. 


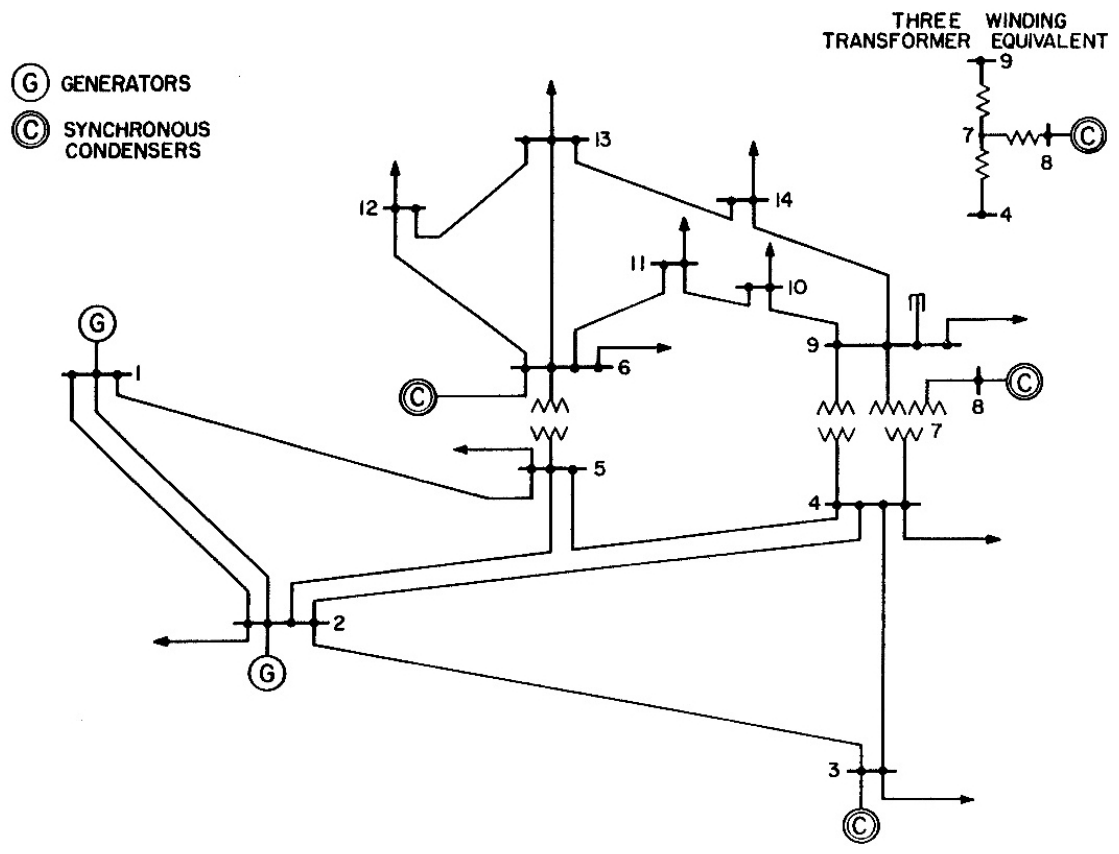

Fig. 4. The IEEE 14-bus test system [15].

\section{B. Results}

Results of the investigations are shown in Table I Table III and in Fig. 5-Fig. 10.

In Table I - Table III, there are gathered results of analysis of parameters of the indices, which describe properties of PSSE for different cases. The mentioned parameters are the minimum, maximum, mean values and standard deviations of the considered indices. Relative changes of parameters of the considered indices for SE_R_S with respect to the suitable parameters of indices for SE_R_S- are given in Table I. Similarly, in Table II, there are relative changes of parameters of the considered indices when SE is realized in the polar coordinate system. The mentioned changes of the parameters of the considered indices characterizing properties of PSSE are calculated using formula: $\Delta p_{X}=100\left(p_{X, S}-p_{X, S-}\right) / p_{X, S-}$, where $p_{X, S}, p_{X, S-}$ are parameters of index $X$, when SE is performed for PS with or without $\mathrm{PhS}$, respectively.

TABLE I. RELATIVE CHANGES OF PARAMETERS OF THE INDICES FOR SE_R_S WITH RESPECT TO SUITABLE PARAMETERS OF INDICES FOR SE R S- (IN PERCENTAGE).

\begin{tabular}{|c|c|c|c|c|c|c|c|c|c|}
\hline \multirow{2}{*}{$\begin{array}{c}\text { number } \\
\text { of MD }\end{array}$} & $\mathbf{m}_{\mathbf{1}}$ & $\mathbf{m}_{\mathbf{2}}$ & $\mathbf{m}_{\mathbf{3}}$ & $\mathbf{m}_{\mathbf{4}}$ & $\mathbf{m}_{\mathbf{1}}$ & $\mathbf{m}_{\mathbf{2}}$ & $\mathbf{m}_{\mathbf{3}}$ & $\mathbf{m}_{\mathbf{4}}$ \\
\hline \multicolumn{8}{|c|}{ number of iterations $\mathbf{n}_{\mathbf{i t}}$} \\
\hline min & 20.0 & 20.0 & 20.0 & 20.0 & 0 & 16.7 & 16.7 & 16.7 \\
\hline max & 14.3 & 14.3 & 16.7 & 16.7 & 37.5 & 12.5 & 14.3 & 14.3 \\
\hline mean & 6.7 & 9.5 & 8.1 & 9.5 & 13.1 & 11.4 & 12.3 & 13.5 \\
\hline std.dev. & 118.2 & 195.2 & 140.8 & 49.2 & 21.5 & -8.7 & -32.3 & -61.1 \\
\hline \multicolumn{8}{|c|}{ condition number cond(G) } \\
\hline min & 108.90 & 93.16 & 92.02 & 90.57 & 111.41 & 88.80 & 82.96 & 74.72 \\
\hline max & 585.87 & 372.98 & 160.98 & 108.06 & 274.11 & 234.57 & 91.80 & 91.69 \\
\hline mean & 260.49 & 132.21 & 107.09 & 96.45 & 254.11 & 129.54 & 104.03 & 92.66 \\
\hline std.dev. & 597.47 & 510.29 & 302.71 & 515.36 & 405.54 & 206.95 & 114.01 & 153.80 \\
\hline \multicolumn{8}{|c|}{ ratio A } \\
\hline min & 7.25 & 5.20 & 0.89 & -4.33 & 11.03 & 5.48 & 7.83 & -3.14 \\
\hline max & 0.90 & 1.17 & 0.17 & 1.49 & 1.20 & 2.02 & 3.79 & 5.42 \\
\hline mean & 3.53 & 1.87 & 0.80 & -0.60 & 4.63 & 5.64 & 6.31 & 7.19 \\
\hline std.dev. & -8.51 & 1.49 & -0.97 & 2.76 & -10.69 & -1.25 & 2.81 & 7.80 \\
\hline
\end{tabular}

Relative differences of the changes of the parameters of the considered indices determined for SE performed in the polar and rectangular coordinate system are shown in Table III. Those relative differences are calculated as $100\left(\Delta p_{\mathrm{X}, \mathrm{P}}-\Delta p_{\mathrm{X}, \mathrm{R}}\right) / \Delta p_{\mathrm{X}, \mathrm{R}}$, where $\Delta p_{\mathrm{X}, \mathrm{P}}, \Delta p_{\mathrm{X}, \mathrm{R}}$ changes of parameter $p_{\mathrm{X}}$, calculated, when $\mathrm{SE}$ is performed in the polar and rectangular coordinate system, respectively.

Analysis of Table I and Table II shows, that the largest differences of the distinguished parameters determined for $\mathrm{SE}$ for PS with $\mathrm{PhS}$ and for PS without $\mathrm{PhS}$ are when condition number cond $(\mathrm{G})$ is taken into account independently of the used coordinate system. Smaller differences of the distinguished parameters are for number of iterations $n_{\text {it }}$ and the smallest ones for ratio $A$.

For all load variants and for all numbers of $\mathrm{MD}$, mean values of condition number cond $(\mathrm{G})$ and number of iterations $n_{\mathrm{it}}$, determined for SE_R_S as well as for SE_P_S are larger than for SE_R_S- or SE_P_S-, respectively. That fact means that the properties of SE_R_S and SE_P_S are worse than the properties of SE_R_S- or SE_P_S-, respectively. The mean values of ratio $A$ are also larger for SE_R_S and SE_P_S than for SE_R_S- or SE_P_S-, respectively, except for load variant $V^{0 . \overline{5}}$ and the MD number equal to $m_{4}$. One can ascertain that in most cases the mean values of ratio $A$ are larger for SE_R_S and SE_P_S than for SE_R_S- or SE_P_S-, respectively, and from the point of view of ratio $A$ the properties of SE_R_S and SE_P_S are worse than the properties of SE_R_S- or SE_P_S-, respectively.

In Fig. 5-Fig. 10, there are presented relative differences of percentiles of appropriate indices for SE_R_S and SE_R_S- or SE_P_S and SE_P_S- as functions of percentile rank, calculated as:

$\Delta P C_{\mathrm{SE} \_\mathrm{R}, \mathrm{X}, \mathrm{r}}=100 \frac{P C_{\mathrm{SE} \_\mathrm{R} \_\mathrm{S}, \mathrm{X}, \mathrm{r}}-P C_{\mathrm{SE} \_\mathrm{R} \_\mathrm{S}-, \mathrm{X}, \mathrm{r}}}{P C_{\mathrm{SE} \_\mathrm{R} \_\mathrm{S}-, \mathrm{X}, \mathrm{r}}}$, 


$$
\Delta P C_{\mathrm{SE} \_\mathrm{P}, \mathrm{X}, \mathrm{r}}=100 \frac{P C_{\mathrm{SE} \_\mathrm{P} \_\mathrm{S}, \mathrm{X}, \mathrm{r}}-P C_{\mathrm{SE} \_\mathrm{P} \_\mathrm{S}-, \mathrm{X}, \mathrm{r}}}{P C_{\mathrm{SE} \_\mathrm{P} \_\mathrm{S}-, \mathrm{X}, \mathrm{r}}},
$$

where $P C_{\mathrm{SE}_{-} \mathrm{R} \_\mathrm{S}, \mathrm{X}, \mathrm{r}}, P C_{\mathrm{SE}_{-} \mathrm{P} \_\mathrm{S}, \mathrm{X}, \mathrm{r}}, P C_{\mathrm{SE}_{-} \mathrm{R} \_ \text {S,-X,r, }}, P C_{\mathrm{SE}_{-} \mathrm{P} \_\mathrm{S}-\mathrm{X}, \mathrm{r}}$ are percentiles of index $X$ of rank $r$ determined for SE_R_S, SE_P_S, SE_R_S-, SE_P_S-, respectively.

TABLE II. RELATIVE CHANGES OF PARAMETERS OF THE INDICES FOR SE P S WITH RESPECT TO SUITABLE PARAMETERS OF INDICES FOR SE_P_S- (IN PERCENTAGE).

\begin{tabular}{|c|c|c|c|c|c|c|c|c|}
\hline \multirow{2}{*}{$\begin{array}{c}\text { number } \\
\text { of } \mathrm{MD}\end{array}$} & \multicolumn{4}{|c|}{ Load variant $V^{0.5}$} & \multicolumn{4}{|c|}{ Load variant $V^{1.5}$} \\
\hline & $\mathrm{m}_{1}$ & $\mathbf{m}_{2}$ & $\mathbf{m}_{3}$ & $\mathbf{m}_{4}$ & $m_{1}$ & $\mathbf{m}_{2}$ & $\mathbf{m}_{3}$ & $\mathbf{m}_{4}$ \\
\hline \multicolumn{9}{|c|}{ number of iterations $n_{\text {it }}$} \\
\hline $\min$ & 60.0 & 60.0 & 60.0 & 60.0 & 50.0 & 50.0 & 50.0 & 33.3 \\
\hline $\max$ & 214.3 & 214.3 & 266.7 & 250.0 & 212.5 & 200.0 & 228.6 & 214.3 \\
\hline mean & 106.3 & 96.9 & 95.0 & 95.2 & 103.9 & 95.4 & 100.4 & 101.4 \\
\hline std.dev. & 719.3 & 1299 & 1096 & 607.4 & 420.4 & 424.5 & 467.2 & 544.6 \\
\hline \multicolumn{9}{|c|}{ condition number cond(G) } \\
\hline $\min$ & 120.94 & 104.66 & 102.41 & 100.85 & 127.78 & 104.26 & 99.93 & 94.26 \\
\hline $\max$ & 593.40 & 348.78 & 164.42 & 111.32 & 308.22 & 226.96 & 133.72 & 118.15 \\
\hline mean & 261.54 & 136.88 & 114.84 & 106.34 & 292.39 & 154.33 & 129.41 & 110.07 \\
\hline std.dev. & 598.95 & 465.10 & 258.45 & 303.65 & 457.52 & 225.66 & 152.02 & 203.29 \\
\hline \multicolumn{9}{|c|}{ ratio A } \\
\hline $\min$ & 7.54 & 5.15 & 0.79 & -4.30 & 10.70 & 5.27 & 7.74 & -3.28 \\
\hline $\max$ & 0.92 & 1.25 & 0.21 & 1.59 & 1.16 & 2.08 & 3.76 & 5.41 \\
\hline mean & 3.54 & 1.87 & 0.81 & -0.58 & 4.63 & 5.64 & 6.30 & 7.16 \\
\hline std.dev. & -8.47 & 1.48 & -0.99 & 2.83 & -10.60 & -1.20 & 2.81 & 7.86 \\
\hline
\end{tabular}

TABLE III. RELATIVE CHANGES OF PARAMETERS OF THE INDICES DETERMINED FOR SE PERFORMED IN POLAR AND RECTANGULAR COORDINATE SYSTEM (IN PERCENTAGE).

\begin{tabular}{|c|c|c|c|c|c|c|c|c|}
\hline \multirow{2}{*}{$\begin{array}{l}\text { number } \\
\text { of } \mathrm{MD}\end{array}$} & \multicolumn{4}{|c|}{ Load variant $V^{0.5}$} & \multicolumn{4}{|c|}{ Load variant $V^{1.5}$} \\
\hline & $\mathbf{m}_{1}$ & $\mathbf{m}_{2}$ & $\mathbf{m}_{3}$ & $\mathbf{m}_{4}$ & $\mathbf{m}_{1}$ & $\mathbf{m}_{2}$ & $\mathbf{m}_{3}$ & $\mathbf{m}_{4}$ \\
\hline \multicolumn{9}{|c|}{ number of iterations $n_{\text {it }}$} \\
\hline $\min$ & 200.0 & 200.0 & 200.0 & 200.0 & & 199.4 & 199.4 & 99.4 \\
\hline $\max$ & 1398.6 & 1398.6 & 1497.0 & 1397.0 & 466.7 & 1500.0 & 1498.6 & 1398.6 \\
\hline mean & 1486.6 & 920.0 & 1072.8 & 902.1 & 693.1 & 736.8 & 716.3 & 651.1 \\
\hline std.dev. & 508.5 & 565.5 & 678.4 & 1134.6 & 1855.3 & -4979 & -1546 & -991.3 \\
\hline \multicolumn{9}{|c|}{ condition number cond $(G)$} \\
\hline $\min$ & 11.06 & 12.34 & 11.29 & 11.35 & 14.69 & 17.41 & 20.46 & 26.15 \\
\hline $\max$ & 1.28 & -6.49 & 14 & 3.02 & 12.44 & -3.24 & 45.66 & 28.86 \\
\hline mean & 0.40 & 3.53 & 7.24 & 10.25 & 15.06 & 19.14 & 24.40 & 18.79 \\
\hline std.dev. & 0.25 & -8.86 & -14.62 & -41.08 & 12.82 & 9.04 & 33.34 & 32.18 \\
\hline \multicolumn{9}{|c|}{ ratio $A$} \\
\hline $\min$ & 4.00 & -0.96 & -11.24 & -0.69 & -2.99 & -3.83 & -1.15 & 4.46 \\
\hline $\max$ & 2.22 & 6.84 & 23.53 & 6.71 & -3.33 & 2.97 & -0.79 & -0.18 \\
\hline mean & 0.28 & 0.00 & 1.25 & -3.33 & 0.00 & 0.00 & -0.16 & $\begin{array}{l}-0.42 \\
\end{array}$ \\
\hline std.dev. & -0.47 & -0.67 & 2.06 & 2.54 & -0.84 & -4.00 & 0.00 & 0.77 \\
\hline
\end{tabular}

For number of iterations, $\triangle \mathrm{PC}_{\mathrm{SE}_{-} \mathrm{P}, \mathrm{nit}, \mathrm{r}}$ is much larger than

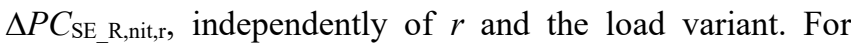
such $r$, for which $\triangle P C_{\mathrm{SE}_{2} \mathrm{R}, \mathrm{nit}, \mathrm{r}}$ is not equal to $0 \%$, $\triangle P C_{\mathrm{SE}_{-} \mathrm{P}, \text { nit,r } \mathrm{r}}$ is from 3 to 15 times larger than $\triangle P C_{\mathrm{SE} \_\mathrm{R}, \mathrm{nit}, \mathrm{r} .}$. For condition number $\operatorname{cond}(\mathbf{G}), \quad \triangle P C_{\mathrm{SE}_{\mathrm{P}}, \operatorname{cond}(\mathbf{G}), \mathrm{r}}$ and $\triangle P C_{\mathrm{SE} \_\mathrm{R}, \text { cond(G), } \mathrm{r}}$ have values which are relatively close to each other. The module of relative difference between these numbers is not larger than $10.6 \%$ for MD number $m_{1}, 9.7 \%$ for MD number $m_{4}$ and load variant $V^{0.5}$ and $7.54 \%$ for MD number $m_{1}, 10.87 \%$ for MD number $m_{4}$ and load variant $V^{1.5}$. Values $\triangle P C_{\mathrm{SE} \_\mathrm{P}, \operatorname{cond}(\mathbf{G}), \mathrm{r}}$ and $\triangle P C_{\mathrm{SE} \_\mathrm{R}, \operatorname{cond}(\mathbf{G}), \mathrm{r}}$ are relatively large. On average, each of them is at least 19 times larger than module of difference between them. Difference between $\triangle P C_{\mathrm{SE}_{\mathrm{P}, \mathrm{A}, \mathrm{r}}}$ and $\triangle P C_{\mathrm{SE}_{\mathrm{R}, \mathrm{A}, \mathrm{r}}}$ is small. On average, the module of it is not larger than $0.09 \% .\left|\triangle P C_{\mathrm{SE}_{\mathrm{P}, \mathrm{A}, \mathrm{r}} \mid}\right|$ and $\left|\triangle P C_{\mathrm{SE} \_\mathrm{R}, \mathrm{A}, \mathrm{r}}\right|$ are not larger than $10 \%$.

\section{DISCUSSION}

Presence of $\mathrm{PhS}$ in PS entails greater complexity of the model of this system. The state vector comprises of larger number of elements. Now, in the state vector are also real and imaginary parts or magnitudes and phase angles of voltages $\overline{\mathbf{V}}_{\text {ET }}$ and $\overline{\mathbf{V}}_{\text {BT }}$, i.e. the number of state variables increases by 4. A number of equations, which are taken into account in SE, increases by 3 . Additional equations result from (10) and (14). Moreover, equations for $P_{i}, Q_{i}, P_{k}, Q_{k}$ where $i$ and $k$ are indices of terminal buses of the branch with $\mathrm{PhS}$, are more complex (see (11)).

From the SE viewpoint, when configuration of measuring systems in PS does not change, situation becomes worse. A level of data redundancy, defined as $r d=n_{d} / n_{s}$, where: $n_{d}$ is a number of data used in a SE process, $n_{s}$ is a number of state variables, decreases. The levels of data redundancy for PS with and without PhS are gathered in Table IV.

TABLE IV. LEVELS OF DATA REDUNDANCY FOR PS WITH AND WITHOUT THE CONSIDERED PHS

\begin{tabular}{|c|c|c|c|c|}
\hline $\mathbf{m}$ & $\mathbf{r d}_{\mathbf{S}-}$ & $\mathbf{r d}$ & $\mathbf{r d}_{\mathbf{S}}-\mathbf{r d}_{\mathbf{S}-}$ & $\begin{array}{c}\left(\mathbf{r d}_{\mathbf{S}}-\mathbf{r d}_{\mathbf{S}-}\right) \mathbf{r d}_{\mathbf{S}} \\
\mathbf{\%}\end{array}$ \\
\hline 34 & 1.172 & 1.121 & -0.051 & -4.37 \\
\hline 53 & 1.828 & 1.697 & -0.131 & -7.15 \\
\hline 68 & 2.345 & 2.152 & -0.193 & -8.24 \\
\hline 104 & 3.586 & 3.242 & -0.344 & -9.59 \\
\hline
\end{tabular}

Note: rds- is a level of data redundancy for PS without PhS, rds is a level of data redundancy for PS with the symmetrical PhS.

Calculating $r d_{s}$ and $r d_{s-}$, where $r d_{s}, r d_{s-}$ are levels of data redundancy for $\mathrm{PS}$ with $\mathrm{PhS}$ and for $\mathrm{PS}$ without $\mathrm{PhS}$, respectively, it is taken into account that in the carried out SE, apart from measurements also virtual measurements are utilized. In this situation, $n_{d}=m+m_{v}$, where $m_{v}$ is a number of the virtual measurements. For PS without $\mathrm{PhS}$ we have $m_{v}=4$, for PS with PhS $m_{v}=7$.

For PS without $\mathrm{PhS}$, the virtual measurements are injections at two zero-injection nodes, i.e. at node 7 and at node 15 . Node 15 is an additional node in the test system. In the investigations, when PS with $\mathrm{PhS}$ is taken into account, the considered $\mathrm{PhS}$ is between nodes 5 and 15 . The power line, which is between nodes 4 and 5 in the original IEEE 14-bus test system, is between nodes 4 and 15 in the modified IEEE 14-bus test system.

For PS with PhS, the virtual measurements are injections at the earlier-mentioned zero-injection nodes, three zero right-hand sides of the equations resulting from relationships (10) (for active and reactive powers) and (14).

The consequence of decreasing the level of data redundancy is possibility of occurrence of larger values of ratio $A \quad\left(A=J_{e} / J_{m}\right)$. Such situation is observed in the performed investigations. Only for certain number of cases, when the load variant is $V^{0.5}$ and the MD number is $m_{4}$, ratio $A$ is larger for SE_R_S- and SE_P_S- than for SE_R_S , and SE_P_S, respectively. However, for the mentioned load variant and the MD number, the relative difference of mean values of $A$ for SE_R_S and SE_R_S- as well as SE_P_S and SE_P_S- is not larger than $0.6 \%$.

Larger number of equations together with greater complexity of some equations and larger number of the 
virtual measurements (having higher accuracy) leads to larger values of condition number $\operatorname{cond}(\mathbf{G})$ and also to worse convergence of the calculation process, i.e. to the larger number of iterations. The results of investigations confirm the presented ascertainment. For load variant $V^{0.5}$ as well as for load variant $V^{1.5}$ and for all considered MD numbers, the mean values and the percentiles of the condition numbers are larger for SE_R_S, and SE_P_S than for SE_R_S- and SE_P_S-, respectively.

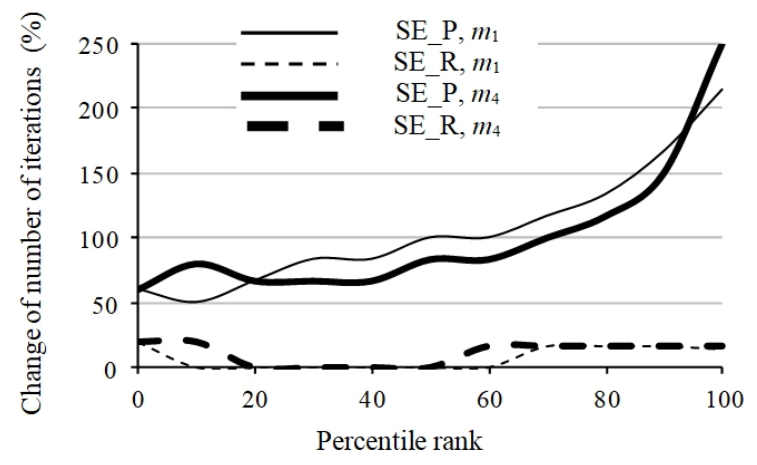

Fig. 5. Relative changes of percentiles of the number of iterations in the $\mathrm{SE}$ process as a function of percentile rank for load variant $V^{0.5}$.

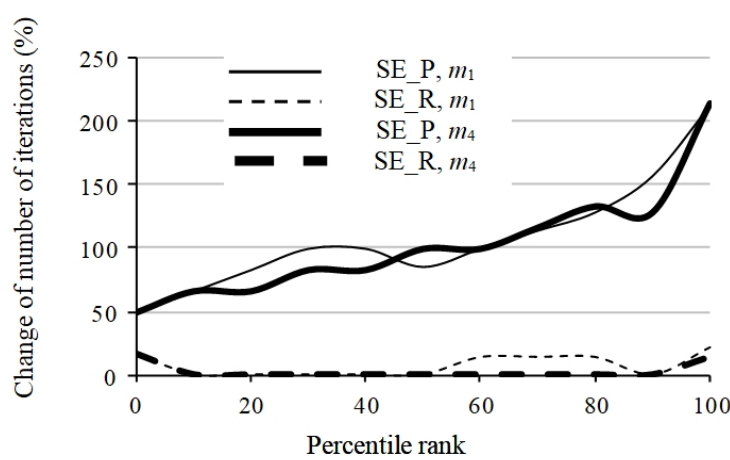

Fig. 6. Relative changes of percentiles of the number of iterations in the SE process as a function of percentile rank for load variant $V^{1.5}$.

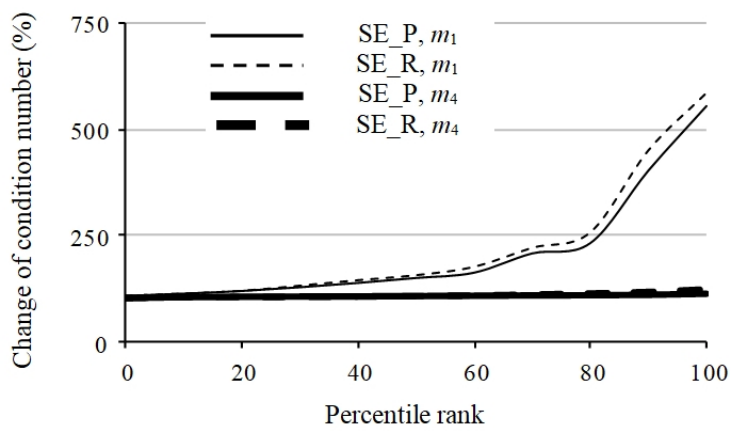

Fig. 7. Relative changes of percentiles of the condition number in the SE process as a function of percentile rank for load variant $V^{0.5}$.

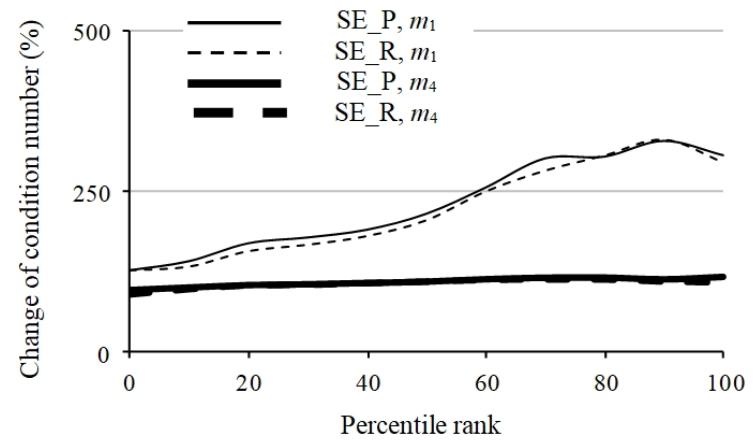

Fig. 8. Relative changes of percentiles of the condition number in the SE process as a function of percentile rank for load variant $V^{I . .5}$.

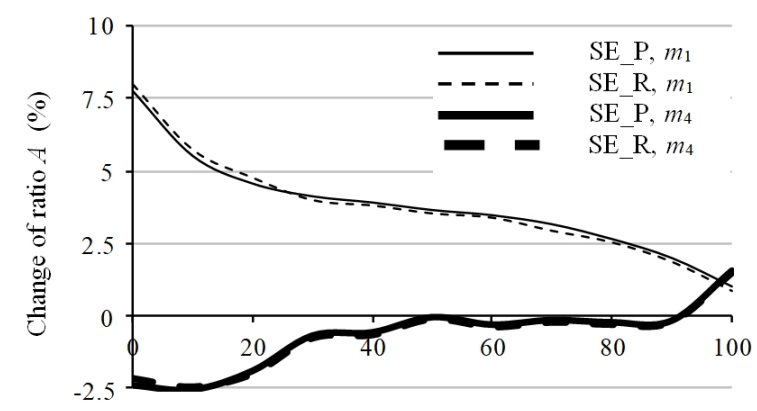

Percentile rank

Fig. 9. Relative changes of percentiles of ratio $A$ in the SE process as a function of percentile rank for load variant $V^{0.5}$.

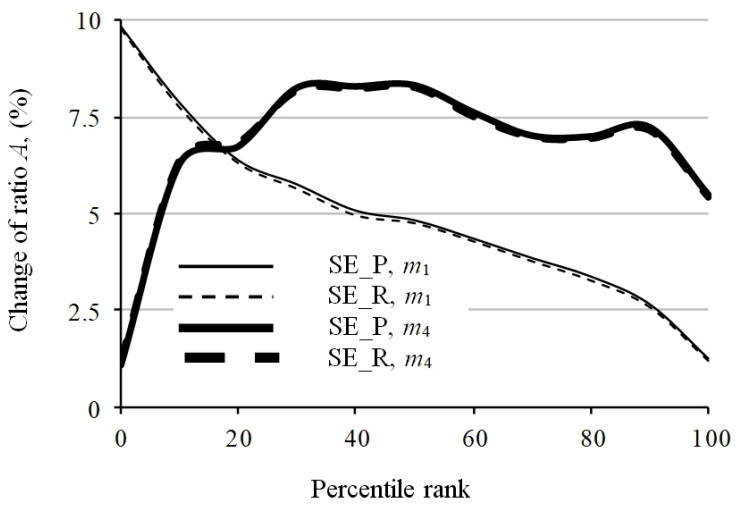

Fig. 10. Relative changes of percentiles of ratio $A$ in the SE process as a function of percentile rank for load variant $V^{1.5}$.

When numbers of iterations are taken into account, one can observe that for PSSE in the rectangular coordinate system for all considered load variants and all considered MD numbers: (i) the mean values of numbers of iterations are larger for SE_R_S than for SE_R_S-, (ii) a certain part of percentiles of numbers of iterations is larger for SE_R_S than for SE_R_S-, and other part of percentiles of numbers of iterations is the same for SE_R_S and for SE_R_S-.

Especially, when the number of iterations is considered, one can note essential influence of the coordinate system on results of PSSE. $\triangle P C_{\mathrm{SE}_{\mathrm{P}}, \mathrm{nit}, \mathrm{r}}$ is essentially larger than $\triangle P C_{\mathrm{SE} \_\mathrm{R}, \mathrm{nit}, \mathrm{r} .}$ That fact can be explained taking into account character of functions, that are in the equations utilized in SE in different coordinate systems. In the rectangular coordinate system, one utilizes equations in which there are quadratic functions, in the polar coordinate system, one utilizes equations in which there are trigonometric functions (that can be considered as infinite series). This fact makes convergence, which is observed in SE calculations in the polar coordinate system, worse.

\section{CONCLUSIONS}

The installation of PhSs in PS changes the conditions that must be taken into account designing SE calculation. In the new conditions, properties of SE are different than it was before. The paper presents original investigations aimed on those properties when in PS there is and there is not one of types of $\mathrm{PhSs}$, which is the symmetrical $\mathrm{PhS}$ (such $\mathrm{PhSs}$ are in Polish Power System). In the paper, one takes into considerations such indices characterizing properties of PSSE as: (i) $n_{i t}-$ the number of iterations of SE calculations, (ii) $\operatorname{cond}(\mathbf{G})$ - the condition number of the gain matrix, that 
plays essential role in the calculations, (iii) ratio $A$ $\left(A=J_{e} / J_{m}\right)$ characterizing accuracy of SE. It is not possible to perform analytical investigation of the mentioned indices in all space of states of PS. In this situation, there were carried out: (i) original calculations of the considered indices for representative operational states of the test PS, (ii) original statistical analyses of the calculated indices, (iii) original discussion on the causes of observed regularities. Analyzing the earlier-mentioned indices, one can ascertain that properties of PSSE are worse when SE is performed for PS with the considered PhS than when it is performed for PS without such the PhS. In each case (defined by the load variant and the data redundancy) mean value and each quantile of number of iterations $n_{i t}$ as well as mean value and each quantile of condition number $\operatorname{cond}(\mathbf{G})$ are larger for SE_R_S or SE_P_S than for SE_R_S-, SE_P_S-, respectively. There are cases, in which the mean value and some quantiles of ratio $A$ are smaller for SE_R_S or SE_P_S than for SE_R_S-, SE_P_S-, respectively. However, number of such cases is relatively low and does not change the earlier-given statement on general evaluation of SE for PS with the considered $\mathrm{PhS}$.

Comparing PSSE for different coordinate systems, one can state, that generally, PSSE has the better properties for the rectangular coordinate system.

The obtained conclusions can be used for seeking a solution to improve the properties of SE for PS with the considered $\mathrm{PhS}$.

\section{REFERENCES}

[1] M. R. Iravani, D. Maratukulam, "Review of semiconductor-controlled (static) phase shifters for power systems applications", IEEE Trans. Power Syst., vol. 9, no. 4, pp. 1833-1839, 1994. DOI: $10.1109 / 59.331438$.

[2] T. Okon, K. Wilkosz, "Phase shifter models for steady state analysis", in Proc. 17th Int. Scientific Conf. on Electric Power Eng. (EPE 2016), 2016, pp. 1-6. DOI: 10.1109/EPE.2016.7521831.

[3] J. Verboomen, D. Van Hertem, P. H. Schavemaker, W. L. Kling,
R. Belmans, "Phase shifting transformers: principles and applications", in Proc. 2005 Int. Conf. on Future Power Systems, 2005, pp. 1-6. DOI: 10.1109/FPS.2005.204302.

[4] C. Schweppe, J. Wildes, "Power system static-state estimation. part IIII", IEEE Trans. Power Appar. \& Syst, vol. 89, no. 1, pp. 120-135, 1970. DOI: 10.1109/TPAS.1970.292680.

[5] N. S. da Silva, A. Simoes Costa, K. A. Clements, E. Andreoli, "Simultaneous estimation of state variables and network topology for power system real-time modelling", Electric Power Systems Research, vol. 133, pp. 338-346, 2016. DOI: 10.1016/j.epsr.2015.12.029.

[6] T. Okon, K. Wilkosz, "WLS state estimation in polar and rectangular coordinate systems for power system with phase shifter", in Proc. Int. Conf. Electr. Power Networks, 2016, pp. 1-6. DOI: 10.1109/EPNET.2016.7999365.

[7] T. Okon, K. Wilkosz, "Modeling quadrature booster in power system state estimation in rectangular coordinate system", in Proc. the 8th Int. Conf. on Energy and Environment (CIEM 2017), Bucharest, Romania, 2017, pp. 1-5. DOI: 10.1109/CIEM.2017.8120841.

[8] E. Acha, C. R. Fuerte-Esquivel, H. Ambriz-Perez, C. AngelesCamacho, FACTS: Modelling and simulation in power networks. Chichester: Wiley, 2004. DOI: 10.1002/0470020164.

[9] M. Arun Bhaskar, C. Subramani, M. Jagdeesh Kumar, S. S. Dash, "Voltage profile improvement using FACTS devices a comparison between SVC, TCSC and TCPST", in Proc. Int. Conf. Advances in Recent Tech. in Communication \& Computing, 2009, pp. 890-892. DOI: 10.1109/ARTCom.2009.135.

[10] T. Okon, K. Wilkosz, "Comparison of weighted-least-squares power system state estimation in polar and rectangular coordinate systems", in Proc. 9th Int. Conf. Environment and Electrical Engg., 2010, pp. 140-143. DOI: 10.1109/EEEIC.2010.5489955.

[11] A. Abur, A. G. Exposito, Power System State Estimation. Theory and Implementation. New York-Basel: Marcel Dekker, Inc., 2004. DOI: 10.1201/9780203913673.

[12] R. Jegatheesan, K. Duraiswamy, "AC:Multi-terminal DC power system state estimation - a sequential approach", Elec. Machines and Power Systems, vol. 12, no. 1, pp. 27-42, 1987. DOI: $10.1080 / 07313568708960093$.

[13] J. Dopazo, O. Klitin, G. Stagg, L. Van Slyck, "State calculation of power systems from line flow measurements", IEEE Trans. Power Appar. \& Syst., vol. PAS-89, no. 7, pp. 1698-1708, 1970. DOI: $10.1109 /$ TPAS.1970.292826.

[14] J. Dopazo, O. Klitin, L. VanSlyck, "State calculation of power systems from line flow measurements, part II", IEEE Trans. Power Appar. \& Syst., vol. PAS-91, no. 1, pp. 145-151, 1972. DOI: 10.1109/TPAS.1972.293324.

[15] Power Systems Test Case Archive. [Online]. Available: http://www.ee.washington.edu/research/pstca/ 